\title{
Venous obstruction in free flap transfer due to severe stenosis of superficial temporal vein
}

\author{
Naohiro Ishii ${ }^{1}$, Takahiro Uno ${ }^{1}$, Tomoki Kiuchi ${ }^{1}$, Kazuo Kishi $^{2}$ \\ ${ }^{1}$ Department of Plastic and Reconstructive Surgery, International University of Health and Welfare Hospital, Nasushiobara; ${ }^{2}$ Department of \\ Plastic and Reconstructive Surgery, Keio University, Tokyo, Japan
}

Small-diameter variation or angiectopia of the superficial temporal vein (STV) has been reported; however, anomalies at its entrance into the parotid gland have not been reported. An 80-year-old man underwent facial reconstruction using a radial forearm free-flap after extensive resection of his right lower eyelid and cheek due to basal cell carcinoma. The procedure's recipient vessels were the superficial temporal artery and two STV (diameter: $2.7 \mathrm{~mm}$ and $1.5 \mathrm{~mm}$ ). Vascular anastomosis successfully performed. The following morning, the transferred flap showed severe congestion (Fig. A). Venous thrombosis occurred $4 \mathrm{~cm}$ from the proximal of the anastomotic site (Fig. B). Vascular reanastomosis alone was unable to resolve the severe venous thrombosis, and the STV outside the parotid gland appeared dilated with severe stenosis at its entrance. However, dissection with the release of the stenosis and further vascular reanastomosis successfully restored venous patency (Fig. C). Fibrous bands causing external compression of the STV were divided. Flap congestion improved immediately and the flap survived completely (Fig. D). The STV entrance into the parotid gland should be examined in both cases of proximal venous thrombosis with satisfactory anastomosis and unexplained dilatation of the dissected STV; preoperative ultrasound may be useful for evaluating the diameter of STV in the latter.

\section{Correspondence: Naohiro Ishii}

Department of Plastic and Reconstructive Surgery, International University of

Health and Welfare Hospital, 537-3 Iguchi, Nasushiobara 329-2763, Japan

E-mail: ishinao0916@gmail.com

Received September 24, 2019 / Revised January 14, 2020 / Accepted February 20, 2020
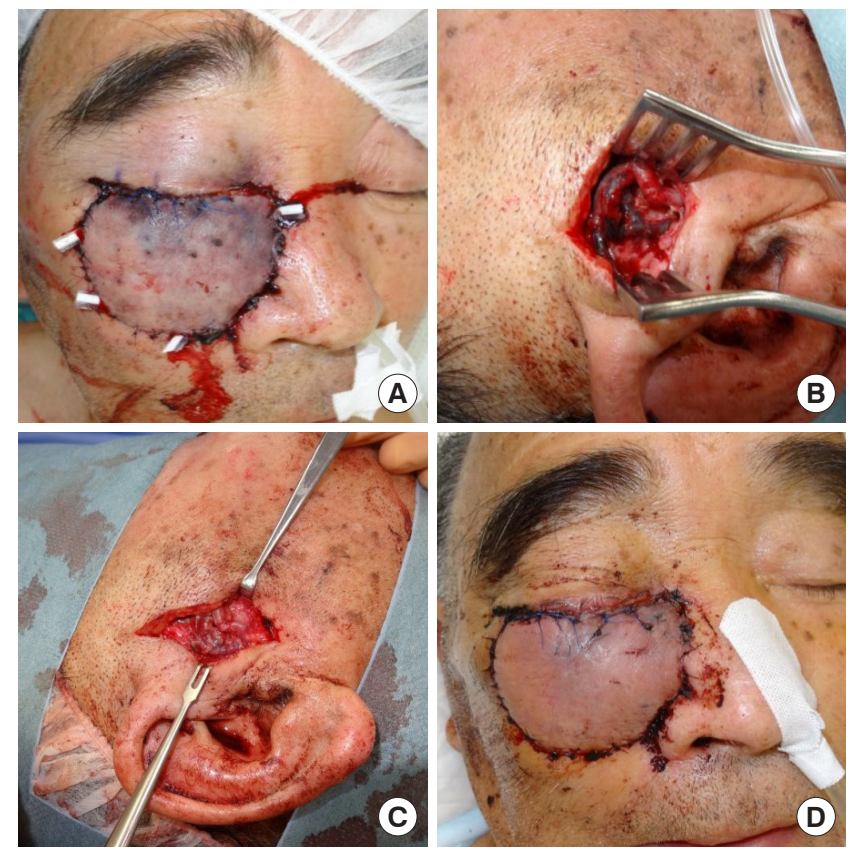

\section{NOTES}

\section{Conflict of interest}

No potential conflict of interest relevant to this article was reported.

\section{Ethical approval}

The study was approved by the Institutional Review Board of Nasu Red Cross Hospital (IRB No. 30-2019-9) and performed in accordance with the principles of the Declaration of Helsinki. Written informed consent was obtained. 


\section{Patient consent}

The patient provided written informed consent for the publication and the use of his images.

\section{ORCID}

Naohiro Ishii

Takahiro Uno

Tomoki Kiuchi

Kazuo Kishi https://orcid.org/0000-0002-0906-5623

https://orcid.org/0000-0002-4170-3701

https://orcid.org/0000-0003-2167-4170

https://orcid.org/0000-0002-4298-9828 